\title{
„Die Coronakrise darf nicht zu einer Krise der Bibliotheken führen“
}

\author{
Der Deutsche Bibliotheksverband fordert finanzielle \\ Unterstützung für Kommunen und ihre Bibliotheken
}

https://doi.org/10.1515/bd-2022-0015

Aufgrund verminderter Steuereinnahmen werden viele Städte und Gemeinden in den kommenden Jahren drastische Kürzungen vornehmen müssen. Wie eine am Dienstag veröffentlichte Studie der Prüfungs- und Beratungsgesellschaft Ernst \& Young (EY) aufzeigt, werden 55 Prozent der insgesamt 304 befragten deutschen Kommunen das laufende Jahr voraussichtlich mit einem Haushaltsdefizit abschließen. 26 Prozent der Städte und Gemeinden planen daher ihre kommunalen Leistungen einzuschränken. Elf Prozent der befragten Kommunen gaben sogar an, Bibliotheken bzw. sonstige kulturelle Einrichtungen schließen zu wollen (Vgl. EY Kommunenstudie 2021: „Kommunen in der Finanzkrise. Status quo und Handlungsoptionen“, https://www.ey.com/de_de/news/2021/12/ey-kommunenstudiedezember-2021).

Dazu Prof. Dr. Andreas Degkwitz, Bundesvorsitzender des Deutschen Bibliotheksverbandes e.V. (dbv): „Die Coronakrise darf nicht zu einer Krise der Bibliotheken werden. Gerade in der jetzigen Situation schaffen Bibliotheken für alle Generationen einen stark nachgefragten Zugang zu Medien, Informationen und zum Internet. Wir appellieren an die neue Bundesregierung, Kommunen und Gemeinden als Träger von Bibliotheken zu entlasten, damit sie auch zukünftig gemeinwohlorientierte Leistungen erbringen können.“

Bereits im Juni 2021 hatte der dbv eine Befragung unter 1.380 Öffentlichen Bibliotheken durchgeführt. Die Ergebnisse: 30 Prozent der an der Befragung teilnehmenden Bibliotheken sind von Maßnahmen der Haushaltskonsolidierung betroffen. 16 Prozent unterliegen einer globalen Haushaltssperre. Bei rund 22 Prozent der Bibliotheken sind bereits Mittel gekürzt worden oder in Planung. Die Ergebnisse der Umfrage sind im „Bericht zur Lage der Bibliotheken 2021“ veröffentlicht worden. Der Bericht kann hier heruntergeladen werden: https://www. bibliotheksverband.de/publikationen. 


\section{Deutscher Bibliotheksverband}

Der Deutsche Bibliotheksverband e.V. (dbv) vertritt mit seinen fast 2.100 Mitgliedern bundesweit über 9.000 Bibliotheken mit 25.000 Beschäftigten und 11 Millionen Nutzer*innen. Sein zentrales Anliegen ist es, Bibliotheken zu stärken, damit sie allen Bürger`innen freien Zugang zu Informationen ermöglichen. Der Verband setzt sich ein für die Entwicklung innovativer Bibliotheksleistungen für Wissenschaft und Gesellschaft. Als politische Interessensvertretung unterstützt der dbv die Bibliotheken insbesondere auf den Feldern Informationskompetenz und Medienbildung, Leseförderung und bei der Ermöglichung kultureller und gesellschaftlicher Teilhabe für alle Bürger*innen.

URL: www.bibliotheksverband.de

\section{Pressekontakt}

Kristin Bäßler

Deutscher Bibliotheksverband

Leitung Kommunikation / Pressesprecherin

Tel.: + 49 (0)30 644989925 | E-Mail: baessler@bibliotheksverband.de 